Journal of Modern Optics

Vol. 00, No. 00, 10 January 2010, 1-8

\title{
Robust High-Fidelity Universal Set of Quantum Gates Through Non-Adiabatic Rapid Passage
}

\author{
Ran $\operatorname{Li}^{a}$ and Frank Gaitan ${ }^{b *}$ \\ ${ }^{a}$ Department of Physics, Kent State University, Stark Campus, North Canton, OH 44720; \\ ${ }^{b}$ Laboratory for Physical Sciences, 8050 Greenmead Dr, College Park, MD 20740
}

()

\begin{abstract}
We show how a robust high-fidelity universal set of quantum gates can be implemented using a single form of non-adiabatic rapid passage whose parameters are optimized to maximize gate fidelity and reward gate robustness. Each gate in the universal set is found to operate with a fidelity $\mathcal{F}$ in the range $0.99988<\mathcal{F}<$ 0.99999 , and to require control parameters with no more than 14-bit (1 part in $\left.10^{4}\right)$ precision. Such precision is within reach of commercially available arbitrary waveform generators, so that an experimental study of this approach to high-fidelity universal quantum control appears feasible.
\end{abstract}

Keywords: fault-tolerant quantum computing; accuracy threshold, quantum interference, group-symmetrized evolution, robust high-fidelity quantum control, non-adiabatic dynamics

\section{Introduction}

Through the accuracy threshold theorem [1 8], it is now known that, under appropriate conditions, an arbitrary quantum computation can be done with arbitrarily small error probability, even in the presence of noise and imperfect quantum gates. The theorem requires the protection of computational data through the use of a suitable quantum error correcting code, and the use of fault-tolerant procedures to control the spread of errors during computation, measurement, and error correction. It also requires the availability of a sufficiently reliable universal set of unencoded quantum gates, where sufficiently reliable means each gate has an error probability $P_{e}$ that is smaller than an accuracy threshold $P_{a}$. The actual value of this threshold is model dependent, though for many, $P_{a} \sim 10^{-4}$ has become a rough-and-ready estimate, with gates anticipated to be approaching the accuracies needed for fault-tolerant quantum computing when $P_{e}<10^{-4}$. The accuracy threshold theorem quantifies the accuracy required of a quantum gate if it is to be used in fault-tolerant quantum computing. However, this is not the only requirement a quantum gate must satisfy. Gate performance must also be robust against small variation of the parameters that specify the control field driving the quantum gate. One of the central challenges facing the field of quantum computing is determining how to implement a robust universal set of unencoded quantum gates for which all gate error probabilities satisfy $P_{e}<10^{-4}$.

In previous work we have shown how controllable quantum interference effects arising during a form of non-adiabatic rapid passage known as twisted rapid passage (TRP) [9 11] can be used to implement a non-adiabatic high-fidelity universal set of quantum gates [12 14]. In Ref. [12], all TRP-generated gates were implemented with error probabilities satisfying $P_{e}<10^{-4}$. Although

${ }^{*}$ Corresponding author. Email: fgaitan@lps.umd.edu 
this level of gate performance achieves the anticipated accuracies needed for fault-tolerant quantum computing, it was found that the performance of the one-qubit gates in the TRP-generated universal set was not sufficiently robust. Specifically, to achieve such high-fidelity performance, the TRP sweep parameters had to be controlled to 1 part in $10^{5}$ (viz. 17-bit) precision. This degree of precision is beyond the capabilities of commercially available arbitrary waveform generators which can only achieve 1 part in $10^{4}$ (14-bit) precision [15]. For TRP to form the basis for high-fidelity universal quantum computation, a way must be found to enhance the robustness of the TRP gates.

In this paper we present a general approach for enhancing the robustness of an arbitrary quantum gate, and apply this approach to the one-qubit gates belonging to the universal set produced using TRP. We show that the resulting gates only require TRP sweep parameters to be controllable to 14-bit precision to operate with error probabilities (fidelities) in the range $6.27 \times 10^{-5}<P_{e}<4.62 \times 10^{-4}(0.99988<\mathcal{F}<0.99999)$. The central result of this paper is that our robustness-enhancing procedure has yielded a universal set of quantum gates that operate with very high fidelity, and yet are sufficiently robustness to be within reach of commercially available arbitrary waveform generators [15]. As we shall see, three (two) of the five TRP gates have error probabilities satisfying $P_{e}<1.12 \times 10^{-4}\left(2.13 \times 10^{-4}<P_{e}<4.62 \times 10^{-4}\right)$, and so operate at (nearly at) the anticipated accuracy needed for fault-tolerant quantum computing.

The structure of this paper is as follows. In Section 2 we briefly review: (i) the necessary background on TRP; (ii) the universal set of quantum gates we implement using TRP; and (iii) the numerical simulations used to determine gate performance. Section 3 then describes the optimization procedure used to maximize gate fidelity while simultaneously rewarding robustness. The results of this optimization procedure are presented in Section 4 for each of the gates in the TRP-generated universal set. Finally, we summarize our results and make closing remarks in Section 5 .

\section{Background}

In an effort to make this paper more self-contained, this Section briefly reviews needed background material on TRP. For a more detailed presentation, the reader is directed to Refs. [9, 12, 14].

\subsection{TRP and Controllable Quantum Interference}

To introduce TRP [9, 14], we consider a single-qubit interacting with an external control-field $\mathbf{F}(t)$ via the Zeeman interaction $H_{z}(t)=-\boldsymbol{\sigma} \cdot \mathbf{F}(t)$, where $\sigma_{i}$ are the Pauli matrices $(i=x, y, z)$. TRP is a generalization of adiabatic rapid passage (ARP) [16]. In ARP, the control-field $\mathbf{F}(t)$ is slowly inverted over a time $T_{0}$ such that $\mathbf{F}(t)=a t \hat{\mathbf{z}}+b \hat{\mathbf{x}}$. In TRP, however, the control-field is allowed to twist in the $x-y$ plane with time-varying azimuthal angle $\phi(t)$, while simultaneously undergoing inversion along the $z$-axis: $\mathbf{F}(t)=a t \hat{\mathbf{z}}+b \cos \phi(t) \hat{\mathbf{x}}+b \sin \phi(t) \hat{\mathbf{y}}$. Here $-T_{0} / 2 \leq t \leq$ $T_{0} / 2$, and throughout this paper, we consider TRP with non-adiabatic inversion. As shown in Ref. [14], the qubit undergoes resonance when

$$
a t-\frac{\hbar}{2} \frac{d \phi}{d t}=0
$$

For polynomial twist, the twist profile $\phi(t)$ takes the form

$$
\phi_{n}(t)=\frac{2}{n} B t^{n}
$$


In this case, Eq. (11) has $n-1$ roots, though only real-valued roots correspond to resonance. Ref. 9] showed that for $n \geq 3$, the qubit undergoes resonance multiple times during a single TRP sweep: (i) for all $n \geq 3$, when $B>0$; and (ii) for odd $n \geq 3$, when $B<0$. For the remainder of this paper we restrict ourselves to $B>0$, and to quartic twist for which $n=4$ in Eq. (2). During quartic twist, the qubit passes through resonance at times $t=0, \pm \sqrt{a / \hbar B}[9]$. It is thus possible to alter the time separating the resonances by varying the TRP sweep parameters $B$ and $a$.

Ref. 9] showed that these multiple resonances have a strong influence on the qubit transition probability, allowing transitions to be strongly enhanced or suppressed through a small variation of the sweep parameters. Ref. [17] calculated the qubit transition amplitude to all orders in the non-adiabatic coupling. The result found there can be re-expressed as the following diagrammatic series:

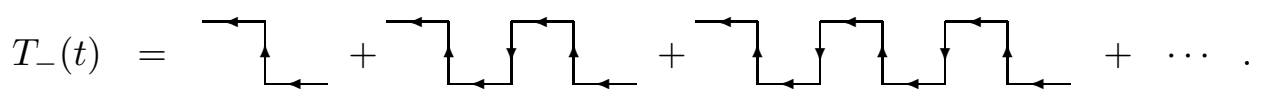

Lower (upper) lines correspond to propagation in the negative (positive) energy-level, and the vertical lines correspond to transitions between the two energy-levels. The calculation sums the probability amplitudes for all interfering alternatives [18] that allow the qubit to end up in the positive energy-level given that it was initially in the negative energy-level. As we have seen, varying the TRP sweep parameters varies the time separating the resonances. This in turn changes the value of each diagram in Eq. (3), and thus alters the interference between the alternative transition pathways. It is the sensitivity of the individual alternatives/diagrams to the time separation of the resonances that allows TRP to manipulate this quantum interference. Zwanziger et al. [10] observed these interference effects in the transition probability using NMR and found quantitative agreement between theory and experiment. It is this link between interfering quantum alternatives and the TRP sweep parameters that we believe underlies the ability of TRP to drive high-fidelity non-adiabatic one- and two-qubit gates.

\subsection{Universal Quantum Gate Set}

The universal set of quantum gates $\mathcal{G}_{U}$ that is of interest here consists of the one-qubit Hadamard and NOT gates, together with variants of the one-qubit $\pi / 8$ and phase gates, and the two-qubit controlled-phase gate. Operator expressions for these gates are: (1) Hadamard: $U_{h}=(1 / \sqrt{2})\left(\sigma_{z}+\sigma_{x}\right)$; (2) NOT: $U_{n o t}=\sigma_{x} ;(3)$ Modified $\pi / 8: V_{\pi / 8}=\cos (\pi / 8) \sigma_{x}-$ $\sin (\pi / 8) \sigma_{y}$; (4) Modified phase: $V_{p}=(1 / \sqrt{2})\left(\sigma_{x}-\sigma_{y}\right)$; and (5) Modified controlled-phase: $V_{c p}=(1 / 2)\left[\left(I^{1}+\sigma_{z}^{1}\right) I^{2}-\left(I^{1}-\sigma_{z}^{1}\right) \sigma_{z}^{2}\right]$. The universality of $\mathcal{G}_{U}$ was demonstrated in Ref. [13] by showing that its gates could construct the well-known universal set comprised of the Hadamard, phase, $\pi / 8$, and CNOT gates.

\subsection{Simulation Procedure}

As is well-known, the Schrodinger dynamics is driven by a Hamiltonian $H(t)$ that causes a unitary transformation $U$ to be applied to an initial quantum state $|\psi\rangle$. In this paper, it is assumed that the Hamiltonian $H(t)$ contains terms that Zeeman-couple each qubit to the TRP control-field $\mathbf{F}(t)$. Assigning values to the TRP sweep parameters $\left(a, b, B, T_{0}\right)$ fixes the control-field $\mathbf{F}(t)$, and in turn, the actual unitary transformation $U_{a}$ applied to $|\psi\rangle$. The task is to find TRP sweep parameter values that produce an applied gate $U_{a}$ that approximates a desired target gate $U_{t}$ sufficiently closely that its error probability (defined below) ideally satisfies $P_{e}<10^{-4}$. In the following, the target gate $U_{t}$ will be one of the gates in the universal set $\mathcal{G}_{U}$. Since $\mathcal{G}_{U}$ contains only one- and two-qubit gates, our simulations will only involve one- and two-qubit systems. 
For the one-qubit simulations, the Hamiltonian $H_{1}(t)$ is the Zeeman Hamiltonian $H_{z}(t)$ introduced in Section 2.1. Ref. [14] showed that it can be written in the following dimensionless form:

$$
\mathcal{H}_{1}(\tau)=(1 / \lambda)\left\{-\tau \sigma_{z}-\cos \phi_{4}(\tau) \sigma_{x}-\sin \phi_{4}(\tau) \sigma_{y}\right\}
$$

Here: $\tau=(a / b) t ; \lambda=\hbar a / b^{2}$; and for quartic twist, $\phi_{4}(\tau)=\left(\eta_{4} / 2 \lambda\right) \tau^{4}$, with $\eta_{4}=\hbar B b^{2} / a^{3}$.

For the two-qubit simulations, the Hamiltonian $H_{2}(t)$ contains terms that Zeeman-couple each qubit to the TRP control-field, and an Ising interaction term that couples the two qubits. Alternative two-qubit interactions can easily be considered, though all simulation results presented below assume an Ising interaction between the qubits. To break a resonance-frequency degeneracy $\omega_{12}=\omega_{34}$ for transitions between, respectively, the ground and first-excited states $\left(E_{1} \leftrightarrow E_{2}\right)$ and the second- and third excited states $\left(E_{3} \leftrightarrow E_{4}\right)$, the term $c_{4}\left|E_{4}(t)\right\rangle\left\langle E_{4}(t)\right|$ was added to $H_{2}(t)$. Combining all of these remarks, we arrive at the following (dimensionless) two-qubit Hamiltonian [13]:

$$
\begin{gathered}
\mathcal{H}_{2}(\tau)=\left[-\left(d_{1}+d_{2}\right) / 2+\tau / \lambda\right] \sigma_{z}^{1}-\left(d_{3} / \lambda\right)\left[\cos \phi_{4} \sigma_{x}^{1}+\sin \phi_{4} \sigma_{y}^{1}\right] \\
+\left[-d_{2} / 2+\tau / \lambda\right] \sigma_{z}^{2}-(1 / \lambda)\left[\cos \phi_{4} \sigma_{x}^{2}+\sin \phi_{4} \sigma_{y}^{2}\right] \\
-\left(\pi d_{4} / 2\right) \sigma_{z}^{1} \sigma_{z}^{2}+c_{4}\left|E_{4}(\tau)\right\rangle\left\langle E_{4}(\tau)\right| .
\end{gathered}
$$

Here: (i) $b_{i}=\hbar \gamma_{i} B_{r f} / 2, \omega_{i}=\gamma_{i} B_{0}, \gamma_{i}$ is the coupling constant for qubit $i$, and $i=1,2$; (ii) $\tau=\left(a / b_{2}\right) t, \lambda=\hbar a / b_{2}^{2}$, and $\eta_{4}=\hbar B b_{2}^{2} / a^{3}$; and (iii) $d_{1}=\left(\omega_{1}-\omega_{2}\right) b_{2} / a, d_{2}=(\Delta / a) b_{2}$, $d_{3}=b_{1} / b_{2}$, and $d_{4}=(J / a) b_{2}$, where $\Delta$ is a detuning parameter [13].

The numerical simulations assign values to the TRP sweep parameters and then integrate the Schrodinger equation to obtain the unitary transformation $U_{a}$ produced by the resulting TRP sweep. Given $U_{a}, U_{t}$, and the initial state $|\psi\rangle$, it is possible to work out [14] the error probability $P_{e}(\psi)$ for the TRP final state $\left|\psi_{a}\right\rangle=U_{a}|\psi\rangle$, relative to the target final state $\left|\psi_{t}\right\rangle=U_{t}|\psi\rangle$. The gate error probability $P_{e}$ is defined to be the worst-case value of $P_{e}(\psi): P_{e} \equiv \max _{|\psi\rangle} P_{e}(\psi)$. Introducing the positive operator $P=\left(U_{a}^{\dagger}-U_{t}^{\dagger}\right)\left(U_{a}-U_{t}\right)$, Ref. [14] showed that the error probability $P_{e}$ satisfies the upper bound $P_{e} \leq \operatorname{Tr} P$. Once $U_{a}$ is known, $\operatorname{Tr} P$ is easily evaluated, and so it is a convenient proxy for $P_{e}$ which is harder to calculate. $\operatorname{Tr} P$ also has the virtue of being directly related to the gate fidelity $\mathcal{F}_{n}=\left(1 / 2^{n}\right) \operatorname{Re}\left[\operatorname{Tr}\left(U_{a}^{\dagger} U_{t}\right)\right]$, where $n$ is the number of qubits acted on by the gate. It is straightforward to show [13] that $\mathcal{F}_{n}=1-\left(1 / 2^{n+1}\right) \operatorname{Tr} P$. The simulations calculate $\operatorname{Tr} P$, which is then used to upper bound the gate error probability $P_{e}$. Note that minimizing $\operatorname{Tr} P$ is equivalent to maximizing the gate fidelity $\mathcal{F}$.

\section{Optimizing Gate Fidelity and Robustness}

To find TRP sweep parameter values that yield highly accurate non-adiabatic quantum gates, it proved necessary to combine the numerical simulations with function minimization algorithms [19] that search for sweep parameter values that minimize the $\operatorname{Tr} P$ upper bound. The multidimensional downhill simplex method was used for the one-qubit gates, while simulated annealing was used for the two-qubit modified controlled-phase gate. This optimization procedure produced one-qubit gates that operate with error probabilities satisfying $P_{e}<10^{-4}[14]$. However, for the two-qubit modified controlled-phase gate $V_{c p}$, simulated annealing was only able to find sweep parameter values that gave $P_{e} \leq 1.27 \times 10^{-3}$ [13]. To further improve the performance of this two-qubit gate, Ref. [12] incorporated the group-symmetrized evolution of Ref. [20] to obtain a modified controlled-phase gate with $P_{e}<10^{-4}$. Group-symmetrized evolution is a form of 
dynamical decoupling that produces an effective dynamics that is invariant under the action of a finite symmetry group $\mathcal{G}$. Ref. [12] identified the finite group $\mathcal{G}$ with the symmetry group of $V_{c p}$, and then used the procedure of Ref. [20] to filter out the $\mathcal{G}$-noninvariant part of the TRP dynamics. As the $\mathcal{G}$-noninvariant dynamics is manifestly bad dynamics relative to $V_{c p}$, groupsymmetrized TRP yields a better approximation to $V_{c p}$, and produces a smaller gate error probability. The reader is directed to Ref. [12] for an detailed explanation of how group-symmetrized evolution is incorporated into a TRP sweep. As noted in Section 1, although this combination of simulation, optimization, and group-symmetrized evolution yielded a universal set of quantum gates that operate with the anticipated accuracy needed for fault-tolerant quantum computing, it was found that the one-qubit gates in $\mathcal{G}_{U}$ could only achieve this level of accuracy if the TRP sweep parameters were controllable to 17-bit $\left(1\right.$ part in $\left.10^{5}\right)$ precision. Such precision is beyond the capabilities of present-day commercially available arbitrary waveform generators. Thus, if TRP is to provide a viable approach to high-fidelity universal quantum control, a way must be found to improve the robustness of the TRP-generated one-qubit gates. In this Section we show how to modify our optimization procedure so that it rewards robust gate operation, while still minimizing (maximizing) $\operatorname{Tr} P$ (gate fidelity).

The optimization procedure just described searches for parameter values $x=\left(x_{1}, \ldots, x_{n}\right)$ that minimize the cost function

$$
\kappa(x)=\operatorname{Tr} P\left(x_{1}, \ldots, x_{n}\right) .
$$

As described in Section 2.3, for the one-qubit gates in $\mathcal{G}_{U}, n=2$, and $x_{1}=\lambda$ and $x_{2}=\eta_{4}$. For $V_{c p}, \operatorname{Tr} P$ depends on seven parameters $\left(\lambda, \eta_{4}, c_{4}, d_{1}, \ldots, d_{4}\right)$. However, for group-symmetrized TRP, only $c_{4}$ and $d_{4}$ are critical parameters [12] and so, effectively, $n=2$ and $\left(x_{1}=c_{4}, x_{2}=d_{4}\right)$. Thus $n=2$ in the cases of interest, although we will consider arbitrary $n$ in the following analysis.

Let $\bar{x}=\left(\bar{x}_{1}, \ldots, \bar{x}_{n}\right)$ and $\bar{\kappa}=\kappa(\bar{x})$ denote the parameter and cost function values, respectively, at an optimization minimum. Suppose we vary the parameters slightly away from $\bar{x}: x_{i}=$ $\bar{x}_{i}+\delta x_{i}(i=1, \ldots, n)$. Taylor-series expanding the cost function $\kappa(x)$ about $\bar{x}$ gives

$$
\kappa(\bar{x}+\delta x)=\bar{\kappa}+\delta^{2} \kappa+\mathcal{O}\left(\delta^{3} x\right)
$$

where the first-order variation vanishes since $\bar{x}$ specifies a minimum of $\kappa(x)$. In Eq. (7),

$$
\delta^{2} \kappa=\frac{1}{2} \sum_{i, j=1}^{n} \delta \xi_{i} H_{i j} \delta \xi_{j},
$$

where $\xi_{i}=x_{i} / \bar{x}_{i}$, and $H$ is the Hessian of the cost function $\kappa$ whose matrix elements are:

$$
H_{i j}=\left.\frac{\partial^{2} \kappa}{\partial \xi_{i} \partial \xi_{j}}\right|_{x=\bar{x}}
$$

It follows from Eq. (7) that the Hessian $H$ determines how rapidly the cost function $\kappa=\operatorname{Tr} P$ varies in the vicinity of the minimum $\bar{x}$. It will thus play an central role in our robustness analysis.

Examination of the Tables in Ref. [13] shows that $\delta^{2} \kappa \sim(30-100) \bar{\kappa}$ when $\bar{x}_{i}$ is varied in its fifth significant figure. As noted earlier, more robust gate performance is desired. Experimental control of the TRP sweep parameters to four significant figures (14-bit precision) is possible and so we can consider a gate to have robust performance if $\delta^{2} \kappa \sim \bar{\kappa}$ when $\bar{x}_{i}$ is varied in its fourth significant figure. This condition can be transformed into a condition on the $l_{1}$-norm [21] of the 
Table 1. Simulation results for the one-qubit gates in $\mathcal{G}_{U}$. The error probability for each gate satisfies $P_{e} \leq \operatorname{Tr} P$.

\begin{tabular}{|c|c|c|c|c|}
\hline Gate & $\lambda$ & $\eta_{4}$ & $\operatorname{Tr} P$ & $\mathcal{F}$ \\
\hline NOT & 6.965 & $2.189 \times 10^{-4}$ & $6.27 \times 10^{-5}$ & 0.99998 \\
Hadamard & 7.820 & $1.792 \times 10^{-4}$ & $1.12 \times 10^{-4}$ & 0.99997 \\
Modified $\pi / 8$ & 8.465 & $1.675 \times 10^{-4}$ & $2.13 \times 10^{-4}$ & 0.99995 \\
Modified phase & 8.073 & $1.666 \times 10^{-4}$ & $4.62 \times 10^{-4}$ & 0.99988 \\
\hline
\end{tabular}

Hessian $H:|| H \|_{1}=\sum_{i, j=1}^{n}\left|H_{i j}\right|$. To see this, suppose that to four significant figure precision, $\bar{x}_{i}=\bar{x}_{i}^{0} \cdot \bar{x}_{i}^{1} \bar{x}_{i}^{2} \bar{x}_{i}^{3} \times 10^{e_{i}}$. Varying $\bar{x}_{i}$ in its fourth significant figure $\left(\bar{x}_{i} \rightarrow \bar{x}_{i}+0.001 \times 10^{e_{i}}\right)$ gives

$$
\delta \xi_{i}=\frac{\delta x_{i}}{\bar{x}_{i}}=\frac{0.001}{\bar{x}_{i}^{0} \cdot \bar{x}_{i}^{1} \bar{x}_{i}^{2} \bar{x}_{i}^{3}}
$$

Combining $\delta^{2} \kappa \sim \bar{\kappa}$ with Eqs. (8) and (10) gives

$$
\bar{\kappa}=\left(5 \times 10^{-7}\right) \sum_{i, j=1}^{n} \frac{H_{i j}}{\left(\bar{x}_{i}^{0} \cdot \bar{x}_{i}^{1} \bar{x}_{i}^{2} \bar{x}_{i}^{3}\right)\left(\bar{x}_{j}^{0} \cdot \bar{x}_{j}^{1} \bar{x}_{j}^{2} \bar{x}_{i}^{3}\right)} .
$$

To arrive at a representative robustness condition, note that for the gate simulations presented in Ref. [12], $\bar{\kappa} \sim 5 \times 10^{-5}$, and $\bar{x}_{i}^{0} \cdot \bar{x}_{i}^{1} \bar{x}_{i}^{2} \bar{x}_{i}^{3} \sim 5$ for all $i$. For such representative values, Eq. (11) gives $\sum_{i, j=1}^{n} H_{i j}=2500$. Noting that $\|H\|_{1} \geq \sum_{i, j=1}^{n} H_{i j}$, this gives $\|H\|_{1} \geq 2500$. Thus robust gate performance will be obtained if the $l_{1}$-norm of the Hessian $H$ satisfies $\|H\|_{1} \sim 2500$. This condition allows us to introduce a penalty function $\mathcal{P}(x)$ for gate robustness, where $\mathcal{P}(x)=$ $\left(\|H\|_{1}-2500\right)^{2}$ when $\|H\|_{1} \geq 2500$; and is zero otherwise. The penalty function $\mathcal{P}(x)$ is small when a gate is operating robustly, and increases as gate performance becomes progressively less robust. To sensitize our optimization procedure to gate robustness, we add $\mathcal{P}(x)$ to the cost function $\kappa(x)$ :

$$
\kappa(x)=\operatorname{Tr} P(x)+r \mathcal{P}(x) .
$$

Here $r$ is a parameter that specifies how heavily gate robustness is weighted during the sweep parameter optimization. When $r=0, \kappa(x)$ reverts to our previous cost function (Eq. (6) $)$ ), and to a robustness-insensitive optimization. In the following Section we use the new cost function (Eq. (12)) to harden the robustness of the one-qubit gates in the universal set $\mathcal{G}_{U}$.

\section{Gate Results}

One-Qubit Gates: Here we present our simulation results for the one-qubit gates in $\mathcal{G}_{U}$, with the sweep parameter optimization based on the new cost function $\kappa(x)$ appearing in Eq. (12). Table 1 gives the optimum values for the dimensionless sweep parameters $\lambda$ and $\eta_{4}$ for each of the onequbit gates in $\mathcal{G}_{U}$. The connection between the dimensionless and dimensionful sweep parameters appears below Eq. (4). Note that all one-qubit simulations were done with dimensionless inversion time $\tau_{0}=80.000$. Table 1 also gives the $\operatorname{Tr} P$ upper bound on the gate error probability $P_{e} \leq$ $\operatorname{Tr} P$ and the gate fidelity $\mathcal{F}$. We see that all one-qubit gates operate with very high-fidelity, with two gates operating with gate error probabilities satisfying $P_{e} \leq 1.12 \times 10^{-4}$, and the remaining two gates satisfying $2.13 \times 10^{-4} \leq P_{e} \leq 4.62 \times 10^{-4}$. Thus all one-qubit gates in $\mathcal{G}_{U}$ operate at, or nearly at, the anticipated accuracy needed for fault-tolerant quantum computing. We now show that, due to our modified optimization procedure, the one-qubit gates are able to achieve the performance given in Table 1 if the TRP sweep parameters are controllable to 14-bit (1 part in $10^{4}$ ) precision. Table 2 shows how $\operatorname{Tr} P$ varies for the Hadamard gate as we vary either $\lambda$ 


Table 2. Sensitivity of $\operatorname{Tr} P$ to small variation of $\lambda$ and $\eta_{4}$ for the one-qubit
Hadamard gate. The three left-most (right-most) columns correspond to varying $\lambda$
$\left(\eta_{4}\right)$ in its fourth significant digit at fixed $\eta_{4}(\lambda)$.
\begin{tabular}{|cccc|ccc|}
\hline$\eta_{4}$ & $\lambda$ & $\operatorname{Tr} P$ & $\lambda$ & $\eta_{4}$ & $\operatorname{Tr} P$ \\
\hline $1.792 \times 10^{-4}$ & 7.819 & $8.05 \times 10^{-4}$ & 7.820 & $1.791 \times 10^{-4}$ & $2.86 \times 10^{-2}$ \\
$1.792 \times 10^{-4}$ & 7.820 & $1.12 \times 10^{-4}$ & 7.820 & $1.792 \times 10^{-4}$ & $1.12 \times 10^{-4}$ \\
$1.792 \times 10^{-4}$ & 7.821 & $2.07 \times 10^{-3}$ & 7.820 & $1.793 \times 10^{-4}$ & $3.11 \times 10^{-2}$ \\
\hline
\end{tabular}

or $\eta_{4}$ in its fourth significant digit. Similar behavior occurs with the other one-qubit gates in $\mathcal{G}_{U}$ and so we do not display corresponding Tables for these gates. Note that when a hardware parameter $p$ is said to have 14-bit precision, it means that it is specifiable to 4 significant figures. Specifically, if one wants $\eta_{4}=1.792 \times 10^{-4}$, the hardware gives that value and not $1.791 \times 10^{-4}$ or $1.793 \times 10^{-4}$. Thus with 14 -bit precision sweep parameters one can hit the optimum sweep parameter values and achieve the performance shown in Table 1. However, with less than 14-bit precision, gate performance will be washed out over the entries in the $\operatorname{Tr} P$ columns of Table 2, and gate performance will not reach the level of Table 1. This is to be compared with Ref. [12] where gates with $P_{e}<10^{-4}$ required sweep parameters with 17-bit precision. The price paid for this enhanced robustness is a slight loss in gate fidelity compared to the one-qubit gates presented in Ref. [12].

Modified Controlled-Phase Gate: As shown in Ref. [12], group-symmetrized TRP is able to produce a two-qubit modified controlled-phase gate $V_{c p}$ which has $\operatorname{Tr} P=8.87 \times 10^{-5}$, corresponding gate fidelity $\mathcal{F}=0.99999$, and error probability satisfying $P_{e} \leq 8.87 \times 10^{-5}$. Ref. [12] also showed that this level of accuracy could be achieved with control parameters specified with 14-bit (1 part in $10^{4}$ ) precision. Thus $V_{c p}$ already operates at the anticipated accuracy needed for fault-tolerant quantum computing with precision demands that are within reach of currently available arbitrary waveform generators. There is thus no need to re-do the optimization of this gate since it is already both sufficiently accurate and robust. The sweep parameter values found in Ref. 12] that produce this gate are included here for completeness: $\lambda=5.04, \eta_{4}=3.0 \times 10^{-4}$, $\tau_{0}=120.00, c_{4}=2.173, d_{1}=99.3, d_{2}=0.0, d_{3}=-0.41$, and $d_{4}=0.8347$.

\section{Discussion}

We have presented a general approach for enhancing the robustness of an arbitrary quantum gate and have applied this approach to the one-qubit gates implemented using twisted rapid passage (TRP). We have shown that the resulting gates operate with error probabilities (fidelities) in the range $6.27 \times 10^{-5}<P_{e}<4.62 \times 10^{-4}(0.99988<\mathcal{F}<0.99999)$, while only requiring TRP sweep parameters that are controllable to 14 -bit (1 part in $\left.10^{4}\right)$ precision. In conjunction with the group-symmetrized two-qubit gate presented in Ref. [12], our robustness enhancing procedure has yielded a universal set of quantum gates that: (i) operate with fidelities that are at, or nearly at, the anticipated accuracies needed for fault-tolerant quantum computing; and (ii) are sufficiently robust to be within reach of commercially available arbitrary waveform generators (AWG) [15]. These results suggest the feasibility of an experimental study (see below) of TRP-based high-fidelity universal quantum control.

In Ref. [12] we showed how TRP could be used to produce a universal set of quantum gates that operate with error probabilities (fidelities) satisfying $P_{e}<10^{-4}(\mathcal{F}>0.9999)$. However, it was found that the one-qubit gates in this universal set required the TRP sweep parameters to be controllable to 17 -bit ( 1 part in $10^{5}$ ) precision which is beyond the reach of commercially available AWG. Using the new optimization procedure presented in Section 3, we have been able to increase the robustness of the one-qubit TRP gates to 14-bit precision which, as noted above, is within reach of commercially available AWG [15]. The cost of this improvement in gate robustness, however, was a slight reduction in the fidelity of the one-qubit TRP gates. 
It is worth noting that earlier work $[9,12-14]$ showed how TRP sweeps could be applied to NMR, atomic, and superconducting qubits, as well as to spin-based qubits in quantum dots; while Ref. [14] described how quantum state tomography could be used to test the performance of the TRP-generated universal quantum gate set. The reader is directed to those papers for further discussion.

A number of directions for future work are possible. (1) Possibly the most important at this time is finding a way to improve the accuracy of the TRP one-qubit gates so that all have error probabilities satisfying $P_{e}<10^{-4}$, while still requiring no more than 14-bit precision on the TRP sweep parameters. Work on this is underway. (2) In previous work we have studied a number of forms of polynomial, as well as periodic, twist [22]. To date, we have found that quartic twist provides best all-around performance when it comes to making the gates in $\mathcal{G}_{U}$. We do not at present have arguments to explain why this is so. We have developed a framework for studying the optimal form of the TRP twist profile $\phi(t)$ based on quantum optimal control theory. We plan to examine this important question in future work. (3) Finally, it would be interesting to study the impact of using a non-Ising two-qubit interaction on the performance of the TRP two-qubit gate.

\section{Acknowledgements}

This research was supported in part by the National Science Foundation through TeraGrid computational resources provided by NCAR under grant TG-PHY100038. One of us (F.G.) thanks T. Howell III for continued support.

\section{References}

[1] D. Aharanov and M. Ben-Or, Fault-tolerant computation with constant error, in Proceedings of the Twenty-Ninth ACM Symposium on the Theory of Computing, 176 (1997).

[2] A. Y. Kitaev, Quantum computation algorithms and error correction, Russ. Math. Surv. 52, 1191 (1997).

[3] A. Y. Kitaev, Quantum error correction with imperfect gates, in Quantum Communication, Computing, and Measurement (Plenum Press, New York, 1997), pp. 181-188.

[4] D. Gottesman, Stabilizer codes and quantum error correction, Ph. D. thesis, California Institute of Technology, Pasadena, CA (1997).

[5] E. Knill, R. Laflamme, and W. H. Zurek, Resilient quantum computation, Science 279, 342 (1998)

[6] E. Knill, R. Laflamme, and W. H. Zurek, Resilient quantum computation: error models and thresholds, Proc. R. Soc. Lond. A 454, 365 (1998).

[7] J. Preskill, Reliable quantum computers, Proc. R. Soc. Lond. A 454, 385 (1998).

[8] F. Gaitan, Quantum error correction and fault-tolerant quantum computing (CRC Press, Boca Raton, FL 2008).

[9] F. Gaitan, Temporal interferometry: a mechanism for controlling qubit transitions during twisted rapid passage with a possible application to quantum computing, Phys. Rev. A 68052314 (2003).

[10] J. W. Zwanziger, U. Werner-Zwanziger, and F. Gaitan, Non-adiabatic rapid passage, Chem. Phys. Lett. 375429 (2003).

[11] F. Gaitan, Controlling qubit transitions during non-adiabatic rapid passage through quantum interference, J. Mod. Opt. 51, 2415 (2004).

[12] R. Li and F. Gaitan, High-fidelity universal quantum gates through group-symmetrized rapid passage, Quantum Info. Comp. 10, 936 (2010).

[13] R. Li, M. Hoover, and F. Gaitan, High-fidelity universal set of quantum gates using non-adiabatic rapid passage, Quantum Info. Comp. 9290 (2009).

[14] R. Li, M. Hoover, and F. Gaitan, High-fidelity single-qubit gates using non-adiabatic rapid passage, Quantum Info. Comp. 7594 (2007).

[15] For example, the Tektronix AWG5000B arbitrary waveform generator provides 14-bit vertical resolution.

[16] A. Abragam, Principles of nuclear magnetism (Oxford University Press, New York 1961).

[17] F. Gaitan, Berry's phase in the presence of a non-adiabatic environment with an application to magnetic resonance, J. Mag. Reson. 139 152(1999).

[18] R. P. Feynman and A. R. Hibbs, Quantum mechanics and path integrals, (McGraw-Hill, New York, 1965).

[19] W.H. Press et al., Numerical Recipes (Cambridge University Press, New York 1992).

[20] P. Zanardi, Symmetrizing evolutions, Phys. Lett. A 25877 (1999).

[21] R.A. Horn and C.R. Johnson, Matrix Analysis (Cambridge University Press, New York 1985).

[22] R. Li and F. Gaitan, Controlling qubit transitions through quantum interference during non-adiabatic rapid passage, Optics and Spectroscopy 99, 257 (2005). 